\title{
Multi-scale modeling of aqueous-phase methane diffusion in silicate channels
}

\author{
Tom Pace ${ }^{1, *}$, Hadi Rahmaninejad ${ }^{1, *}$, Bin $\operatorname{Sun}^{2}$, and Peter \\ Kekenes-Huskey ${ }^{2}$ \\ ${ }^{1}$ Department of Physics and Astronomy, University of Kentucky \\ ${ }^{2}$ Department of Cell \& Molecular Physiology, Loyola University \\ Chicago \\ ${ }^{*}$ Corresponding authors
}

May 14, 2021 


\section{Abstract}

Silica-based materials including zeolites are commonly used for wide ranging applications including separations and catalysis. Substrate transport rates in these materials often significantly influence the efficiency of such applications. Two factors that contribute to transport rates include 1) the porosity of the silicate matrix and 2) non-bonding interactions between the diffusing species and the silicate surface. Here, we utilize computer simulation to resolve the relative contribution of these factors to effective methane transport rates in a silicate channel. Specifically, we develop a 'homogenized' model of methane transport valid at micron and longer length scales that incorporates atomistic-scale kinetic information. The atomistic-scale data are obtained from extensive molecular dynamics simulations that yield local diffusion coefficients and potentials of mean force. With this model, we demonstrate how nuances in silicate hydration and silica/methane interactions impact 'macroscale' methane diffusion rates in bulk silicate materials. This hybrid homogenization/molecular dynamics approach will be of general use for describing small molecule transport in materials with detailed molecular interactions.

\section{Introduction}

Porous media are encountered in many applications in a variety of fields of science and engineering, including chemical reactors, groundwater analysis, petroleum extraction, and even some types of artificial organs [1, 2]. In particular, these applications often depend on the rate of transport of a given reactant, contaminant, or other chemical species trough the fluid-filled pores of the material. Such transport properties are often influenced by physical processes at a range of different scales of analysis, beginning with inter-molecular interactions among the solute, solvent, and solid matrix of the system. These interactions can be studied with atomistic analyses such as molecular dynamics (MD) simulations. Phenomena that become relevant at larger scales include the porosity of the medium and the tortuosity of the paths by which the solute can diffuse [2]. At the macroscopic scale, the transport of a solute through a saturated porous medium is well-described by Partial Differential Equations (PDEs) such as the Fickian diffusion equation, treating the porous medium as a continuum. At intermediate scales, the PDE must include the combined effect of diffusion and the net forces resulting on the solute from the smaller-scale interactions. These net forces can be included through a spatially-varying scalar potential, in which case the diffusion equation becomes the Smoluchowski diffusion equation as described in Sect. 6.2.1.

Here, we investigate the possible connections between the models at these different scales of analysis. We propose a multi-scale model incorporating the chemical and physical processes that dominate different portions of the hierarchy of scales. First, we obtain local transport properties and Potential of Mean Forces (PMFs) from atomistic simulations as described in Sect. 5.2. The mathematical procedure of homogenization $[3,4]$ is then used to aggregate the spatial variation of these quantities into an effective transport property for a larger analysis scale. We demonstrate these techniques on an example system consisting of methane as the solute, water as the solvent, and a nanoporous silicate medium. The silicate geometry is shown in Figure 1. A periodic material with this geometry would contain multiple parallel channels.

Our expectations for this system are that spatial variations in the water density at the atomistic scale will govern the local diffusion coefficient and PMF variations, which in turn will affect the effective diffusion coefficient appropriate 
for larger scales. We explored variations of the system including alterations of the porosity and changes to the surface chemistry of the silicate.

Prior studies have investigated hydrocarbon transport in silica-based frameworks. Hansen et al. [5] considered the alkylation of benzene within a zeolite catalyst. The reaction and diffusion were considered in the gas phase. Consequently, the Maxwell-Stefan diffusion equations were used rather than Fickian or Smoluchowski diffusion. Information from MD and kinetic Monte Carlo simulations was used to provide inputs to the continuum diffusion model. No PMF was considered. The continuum diffusion model was evaluated analytically rather than numerically. One key focus of the study was to predict reaction rates agreeing with experiment, which is not attempted herein.

Another similar analysis was conducted by Bui et al. [6], where a channel of one nanometer in width was studied in various materials, including silica. The study computed the diffusion coefficient within the channel using the Mean Squared Displacement (MSD) of unrestrained methane. This results in a coefficient value that does not distinguish spatial variations within the channel. No multi-scaling method was applied to the result. In addition to using a metadynamics approach to evaluate the free energy variations within the channel, umbrella sampling was also applied. Finally, the silica structure of the Bui et al. [6] study does not appear to include silanol terminations at the surface. The silanol groups present on the silicate surfaces studied herein would be expected to increase the affinity for water molecules, leading to different results for the water density within the channel, and consequently affecting the PMF and diffusion coefficients. Similar to the results presented below, the study found anisotropy in the diffusion coefficient, with differing coefficients for directions perpendicular to the silica face than parallel to it. 
a)

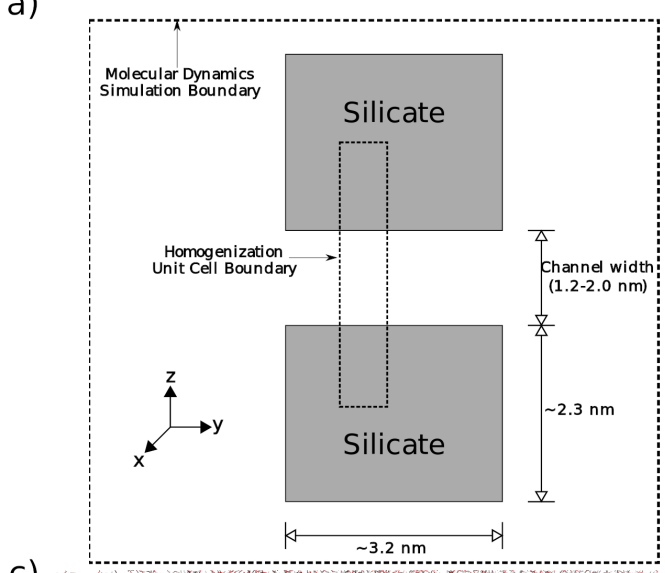

c)

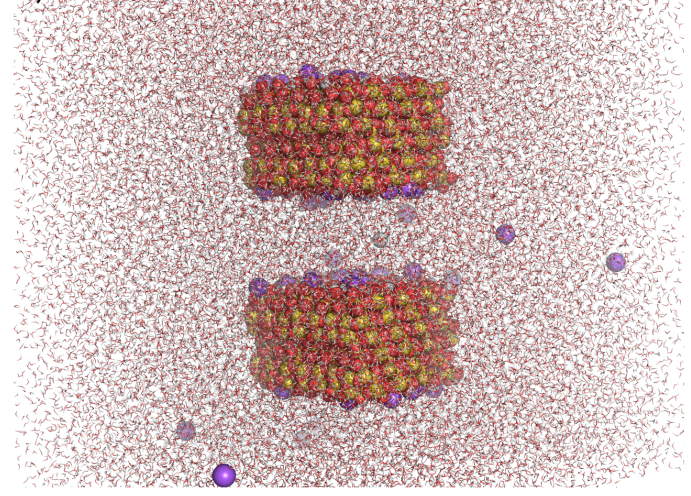

b)
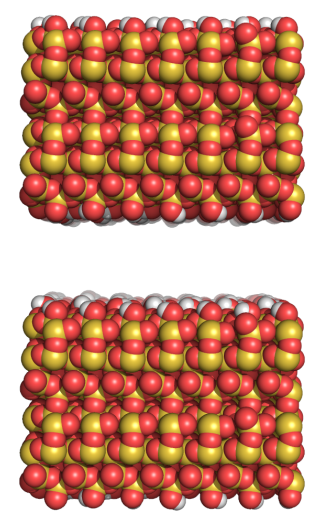

d)

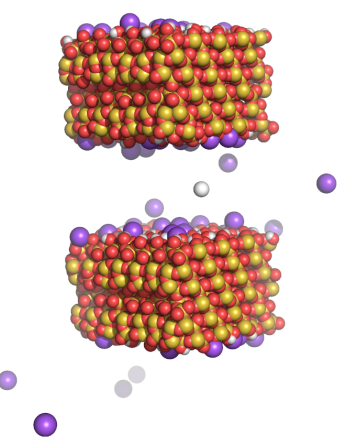

Figure 1: $\quad$ Silicon dioxide $\left(\mathrm{SiO}_{2}\right)$ matrix with 'slit' of variable height (1.2$2.0 \mathrm{~nm}$ ) a) Sketch showing key dimensions, coordinate axes, and simulation boundaries. b) Rendering of the $1.2 \mathrm{~nm}$ channel along the X-axis in the fully protonated condition. c) Oblique rendering of the $2.0 \mathrm{~nm}$ channel with $27 \%$ deprotonation after solvation and equilibration. Sodium $(\mathrm{Na})$ ions are shown as purple spheres. d) Same view as (c) without water molecules. The methane molecule is visible in white, near mid-channel. The silicate structures provided in Emami et al. [7, 8] were used to construct this geometry in the MD simulations. 


\section{Results}

\subsection{Validation of diffusion coefficient extraction procedure in bulk conditions}

MD simulations were conducted to validate the approach presented in Section 5.2 for obtaining the local diffusion coefficient. These validation simulations include a single methane molecule in bulk water, to facilitate comparison with experimental results presented in Witherspoon and Saraf [9].

The validation simulation was conducted with two different force fields: the force field described in Section 6.1.1 derived from Emami et al. [7], and the GROMOS 53A6 force field [10] used by Daldrop, Kowalik, and Netz [11] in their validation. Each simulation was $16 \mathrm{~ns}$ in duration, and was conducted at a temperature of $298 \mathrm{~K}$. Both force fields gave MSD results that approximated the expected result of $k_{B} T / K$, and the two simulation values were in agreement with one another.

An example positional Autocorrelation Function (ACF) curve from each simulation is shown in Figure 2. The diffusion coefficient for methane in bulk water was then calculated in both simulations from the ACF and MSD for each coordinate. The results are shown in Table 1. The GROMOS 53A6 force field matches the experimental results quite well, as reported by Daldrop, Kowalik, and Netz [11]. In contrast, the selected force field overestimates the diffusion coefficient by roughly $60 \%$, because it gives a slightly lower curve for the ACF as shown in Figure 2. However, the selected force field is preferred for the silicate MD simulations in order to properly represent the silicate structure. Accordingly, local diffusion coefficient values computed using the selected force field should be normalized by the bulk value presented here for correct interpretation. Specifically, the local diffusion coefficient values are divided by $3.0 \times 10^{-5} \mathrm{~cm}^{2} / \mathrm{sec}$ to report the ratio of local diffusion to bulk diffusion.

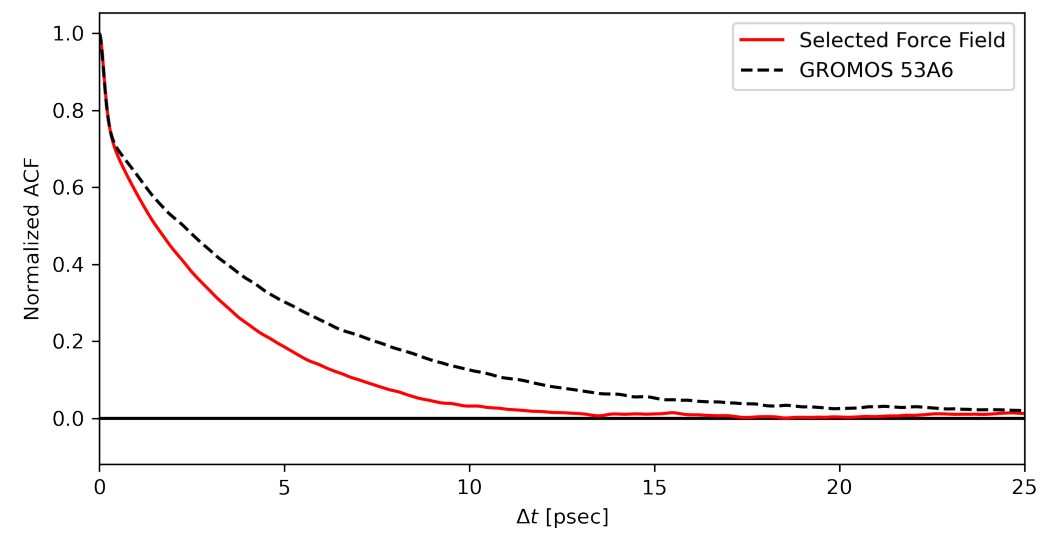

Figure 2: Comparison of position Autocorrelation Functions using two different force fields. The ACF values are normalized to their respective coordinate MSD values. The selected force field is described in Section 6.1.1. Both curves are for the z-direction. The results from the GROMOS 53A6 force field provide a diffusion coefficient that matches the result from physical measurement. 
Table 1: Diffusion coefficient results from simulations of methane in bulk water.

\begin{tabular}{cccc} 
Direction & $\begin{array}{c}\text { Experimental } \\
\text { Result [9] } \\
\left(1 \times 10^{-5} \mathrm{~cm}^{2} / \mathrm{sec}\right)\end{array}$ & $\begin{array}{c}\text { Selected } \\
\text { Force Field } \\
\left(1 \times 10^{-5} \mathrm{~cm}^{2} / \mathrm{sec}\right)\end{array}$ & $\begin{array}{c}\text { GROMOS 53A6 } \\
\text { Force Field } \\
\left(1 \times 10^{-5} \mathrm{~cm}^{2} / \mathrm{sec}\right)\end{array}$ \\
\hline $\mathrm{x}$ & 1.88 & 3.13 & 1.91 \\
$\mathrm{y}$ & 1.88 & 2.96 & 1.73 \\
$\mathrm{z}$ & 1.88 & 3.06 & 1.85 \\
\hline Mean Value & 1.88 & 3.05 & 0.09 \\
\hline $\begin{array}{c}\text { Sample Standard } \\
\text { Deviation }\end{array}$ & $\mathrm{n} / \mathrm{a}$ & 0.09 & $5 \%$ \\
\hline $\begin{array}{c}\text { Coefficient } \\
\text { of Variation }\end{array}$ & $\mathrm{n} / \mathrm{a}$ & $3 \%$ &
\end{tabular}

As a further validation of the local diffusion coefficient, an alternative method was also used for the simulation of methane in bulk water. In these simulations, the methane was not restrained. As indicated in [12], a diffusing particle without restraint is expected to have a MSD that increases linearly with time. The derivative of the MSD with respect to time is $6 D$. Accordingly, for the simulations of unrestrained methane in bulk water, the MSD was plotted against time and fit to a linear increase, with the diffusion coefficient calculated from this fit. Results from three trials (trial A, trial B, and trial C), are shown in Figure 3. The resulting diffusion coefficients are shown in Table 2. Notably, the MSD approach for unrestrained methane exhibited greater variation in the diffusion coefficient than the ACF approach for restrained methane. 


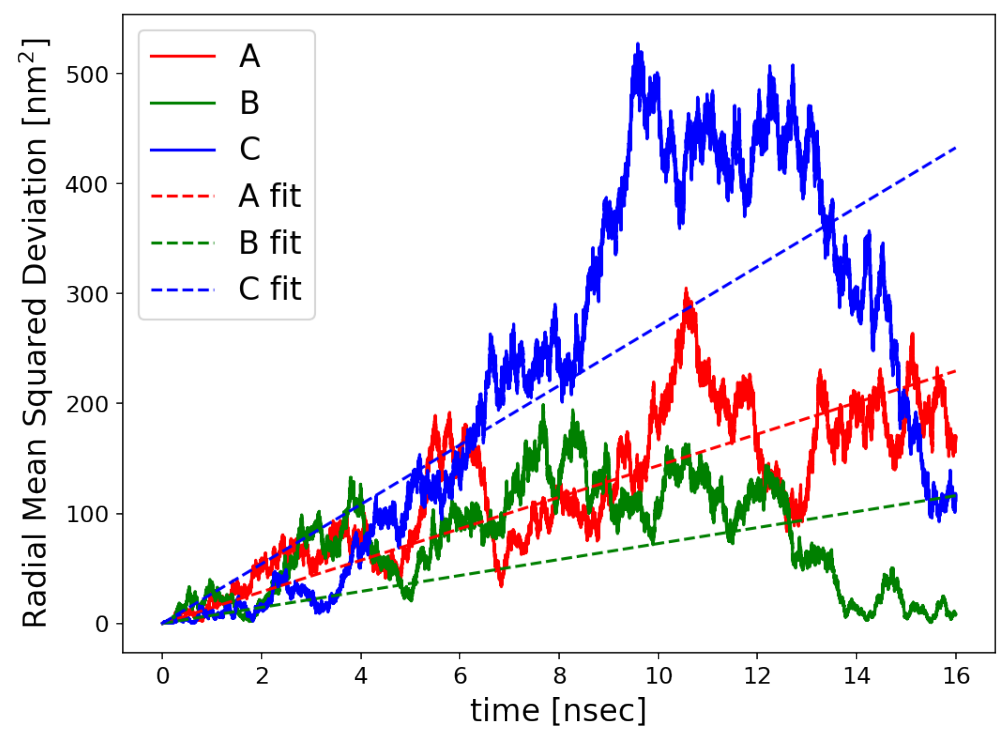

Figure 3: Calculation of diffusion coefficient from MSD, for three different trials. Each simulation had a duration of $16 \mathrm{~ns}$. 
Table 2: Diffusion coefficient results from unrestrained methane simulations in bulk water.

\begin{tabular}{cc} 
Trial & $\begin{array}{c}\text { Result from } \\
\text { MSD } \\
\left(1 \times 10^{-5} \mathrm{~cm}^{2} / \mathrm{sec}\right)\end{array}$ \\
\hline Trial A & 2.39 \\
Trial B & 1.21 \\
Trial C & 4.51 \\
\hline Mean of Trials & 2.70 \\
Sample Standard Deviation & 1.67 \\
Coefficient of Variation & $62 \%$
\end{tabular}

Figure 4 summarizes the validation results by averaging over the directional components. As noted above, the experimental results of Witherspoon and Saraf [9] agreed with the ACF calculation using the GROMOS 53A6 force field. The ACF and MSD approaches using the selected force field gave notably higher diffusion coefficient values, but were in general agreement with one another. Accordingly, the selected force field is assumed to give diffusion coefficients that are proportional, but not identical, to the expected results of experiment. This proportionality is applied to the results by normalizing to the bulk water diffusion result as described above.

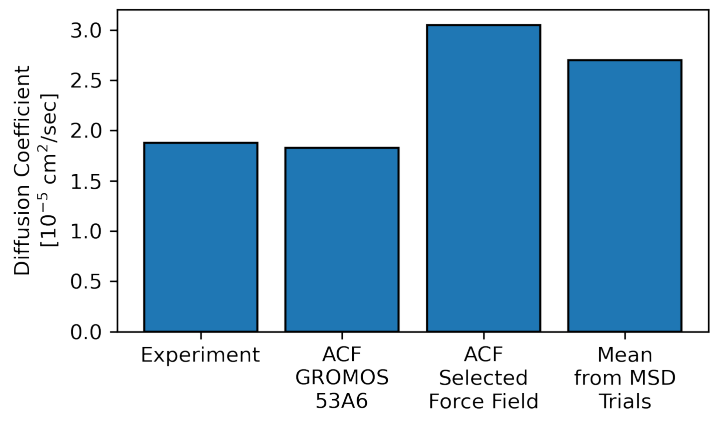

Figure 4: Comparison of diffusion coefficient for methane in bulk water from different methods. 


\subsection{Molecular Dynamics Simulations}

\subsubsection{Results for $1.6 \mathrm{~nm}$ channel}

MD simulations were conducted for the silicate geometry shown in Fig. 1, for a channel width of $1.6 \mathrm{~nm}$. Water density calculations were performed using water trajectories from simulations containing only the silicate and water, in the absence of the methane solvent. The water density within the simulation volume is shown in Fig. 5 for a single plane. A region of the channel containing areas of both high and low water density was selected for further MD simulations to map the spatial variations of both the local diffusion coefficient and the PMF.

Local diffusion coefficients were calculated as described in Sections 5.2 and 6.1.2. Fig. 6 shows the position ACF results for three different locations within the $1.6 \mathrm{~nm}$ channel. The curve for the bulk simulation presented in Section 3.1 (see Figure 2) is also included for comparison. The ACF curve for the bulk condition is from a 16 ns simulation, while the channel simulations were $2 \mathrm{~ns}$ long. This results in a smoother curve for the bulk condition in the figure. Otherwise, the curve that most nearly matches the bulk simulation result is from a point near the middle of the channel. Fig. 6 also shows the local diffusion coefficient results plotted against position within the channel. The local diffusion coefficient appears to approach the bulk value near the middle of the channel, and drop to roughly half this value at positions closer to the channel surfaces. 


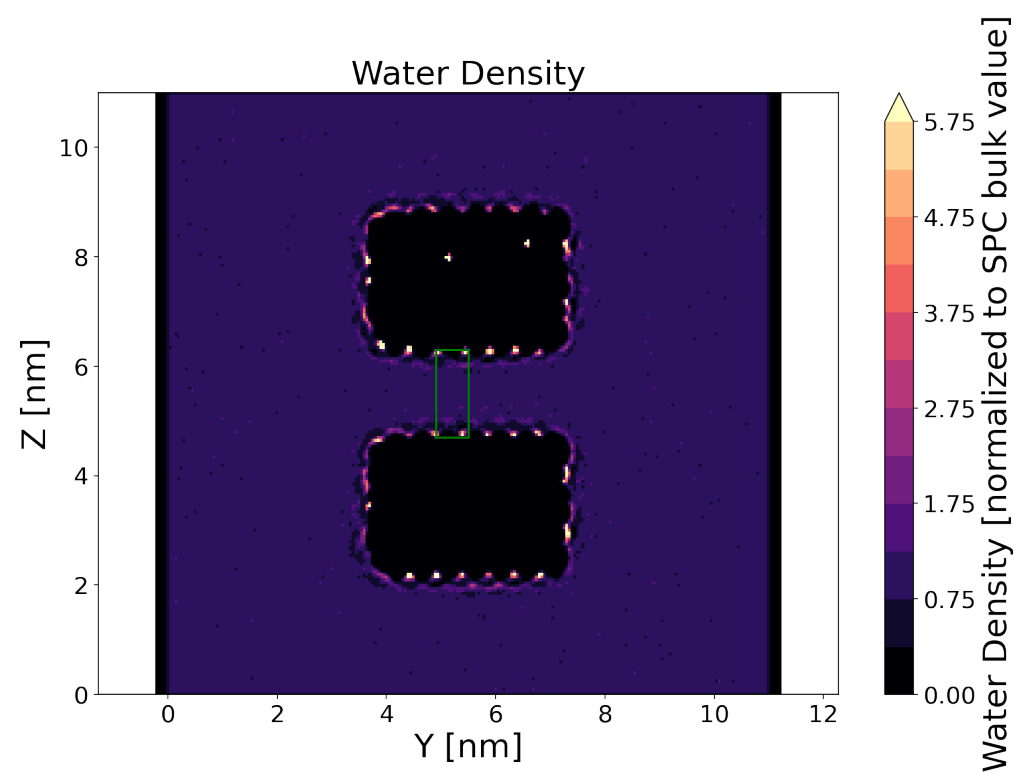

Figure 5: Water density over one plane in the MD simulation for the system shown in Fig. 5, with a $1.6 \mathrm{~nm}$ channel width between the two silicates. The dark areas of near-zero water density are the region occupied by the silicates. Large variations in the water density are observed near the silicate surfaces. The remainder of the model has a water density typical of bulk conditions. The green rectangle highlights the portion of the model selected for detailed measurement of the local diffusion coefficient and PMF. The water density was calculated at half- $\AA$ spatial resolution, from 128 ns of simulation data. 
Fig. 7 illustrates the spatial variation of the PMF, local diffusion coefficient, and water density within the $1.6 \mathrm{~nm}$ channel. The values are averaged in the $y$-direction, to demonstrate the general trend in values across the channel. The local diffusion coefficient seems to be suppressed near the channel walls, and approaches, but does not match, the bulk diffusion behavior near the center of the channel. This variation in the local diffusion coefficient is symmetric about the channel. In contrast, the PMF appears to be asymmetric within the channel, though still suppressed near the surfaces. This asymmetry may be the result of the asymmetry of the silicate faces themselves; the faces are not mirror images of one another. Also, the nearest silanol groups at each face are centered at different distances from the plane of consideration. Some of the asymmetry in the PMF may also be due to noise that could be reduced by longer simulations at each point of measurement. The water density seems to oscillate near the channel walls, likely due to solvation layers around the silicate, but the strength of this oscillation reduces quickly with distance from the surface. These patterns suggest that the local water density, and gradients or other functions thereof, may indeed be a critical driver of variations in the PMF and local diffusion coefficient.
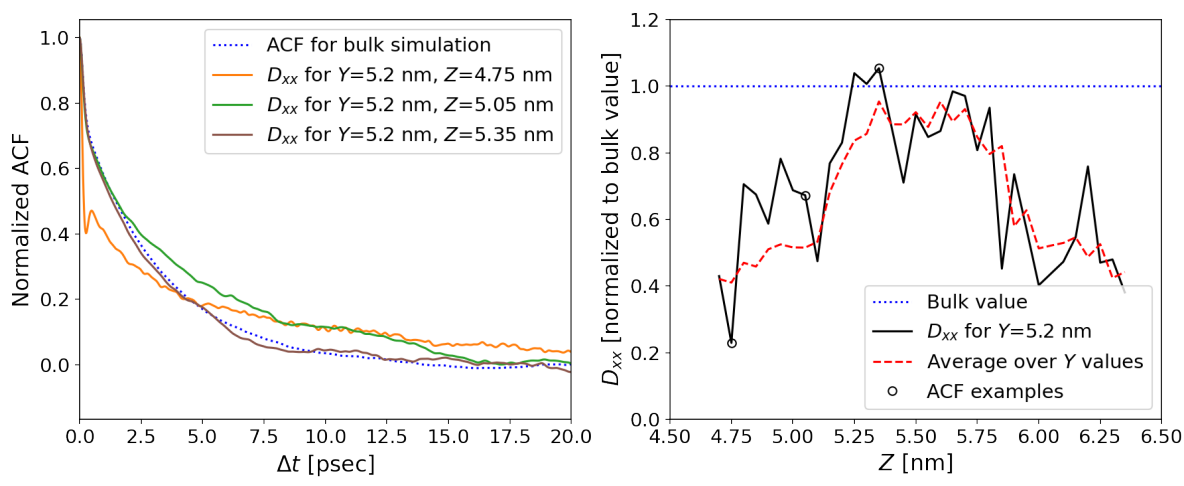

Figure 6: $\quad$ Examples of local diffusion coefficient in the $1.6 \mathrm{~nm}$ channel. Left: Position autocorrelation function of methane for three different positions in the channel. Right: Local diffusion coefficient variation within the channel, showing the values along a line across the channel ( $z$-direction), and also the averages over lines parallel to the channel face ( $y$-direction). The locations that have ACF curves on the left panel are marked by circles on the right panel. The coordinate system is as shown in Figure 1. 
Fig. 8 shows contour plots of the water density, local diffusion coefficient, and potential of mean force from the MD simulations for the $1.6 \mathrm{~nm}$ channel width, illustrating the variations of all three quantities over two spatial dimensions of the channel. The water density data is the same as shown in Fig. 5, but over a smaller region of the plane, centered near the area where measurements of the PMF and local diffusion coefficient were obtained. The water density is near its bulk value over most of the channel, but has some localized areas of high water density at nearly consistent spacing along the channel surfaces. This is most likely due to the presence of hydrophilic silanol groups present on the surface in repeating patterns. The local diffusion coefficient results show the same pattern as the results in Figure 6 and 7: the value is highest near the middle of the channel, where the value is roughly the bulk diffusion coefficient, and then suppressed closer to the channel surfaces. The local diffusion coefficient seems to vary more with distance from the channel wall than with position along the channel. The PMF results are generally highest at the channel walls, and lower in the middle of the channel, although localized depressions in the PMF seem to appear at the channel surface in a generally periodic arrangement. Again, these depressions may be due to the periodic, but asymmetric, pattern of silanol groups on the silicate channel faces.

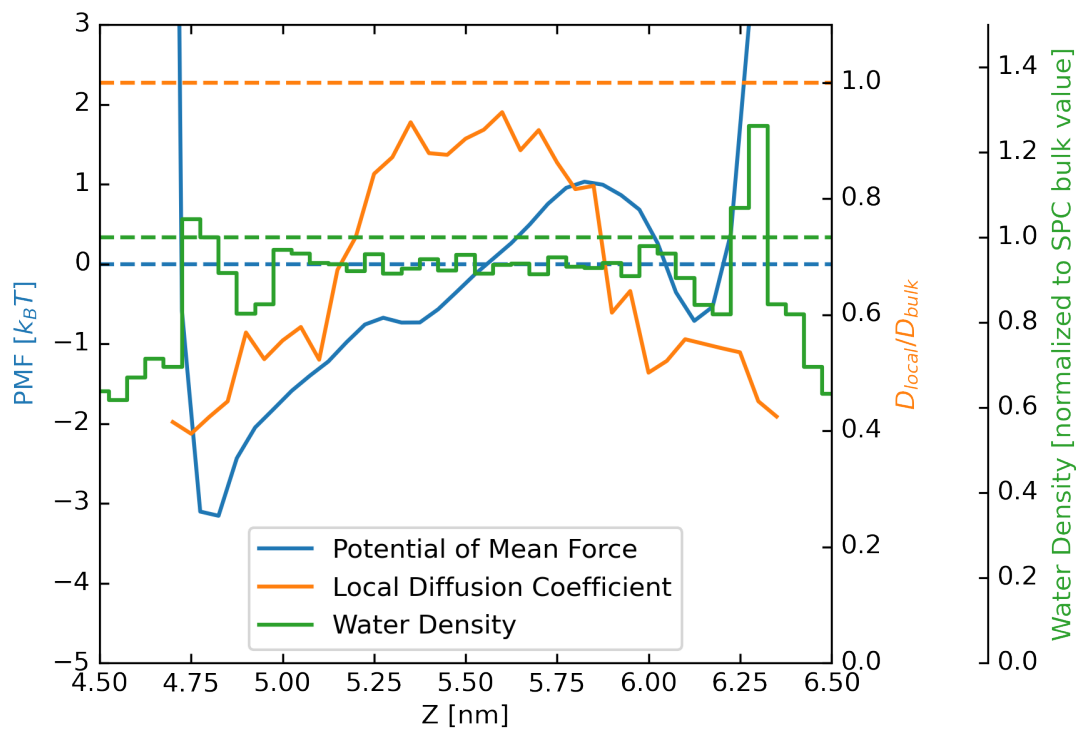

Figure 7: Spatial variation of averaged PMF, local diffusion coefficient, and water density in the $1.6 \mathrm{~nm}$ channel. The values are averaged over $0.6 \mathrm{~nm}$ in the $y$-direction, for a single value of $x$, showing the spatial variation in the $z$-direction (perpendicular to the channel faces). Dashed lines show the values under bulk conditions. 

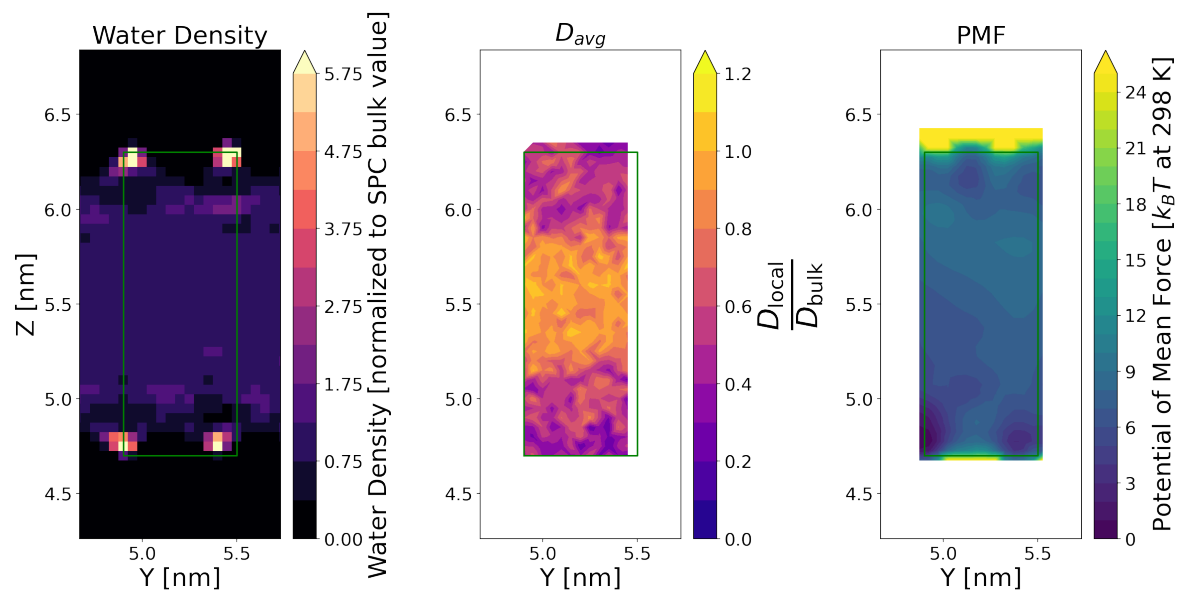

Figure 8: Water density, local diffusion coefficient, and potential of mean force for the $1.6 \mathrm{~nm}$ channel width, fully protonated. The imposed rectangle shows the bounds of the data selected for the Finite Element model within the plane. The water density calculation is from 128 ns of simulation data, at half- $\AA$ spatial resolution. 


\subsubsection{Results for channels of varying porosity}

To assess the influence of porosity on transport behavior within the silicate channel, MD simulations were conducted for channel widths of 1.2, 1.6, and 2.0 $\mathrm{nm}$. Results for water density, PMF, and local diffusion coefficient for these channel widths are shown in Figure 9. The water density follows a similar pattern for all three channel widths: the density is very nearly the value under bulk conditions over most of the channel width, but increases at select locations near the silicate surface. The oscillations of the water density are somewhat indicative of solvation shells around the silicate. The PMF results generally follow a pattern of approximating the bulk value near the middle of the channel, though localized areas of low PMF adjacent to the silicate surface are sometimes present. The local diffusion coefficients also follow a similar pattern in all three channel widths: the coefficient is highest near the middle of the channel, and drops to a lower value at locations closer to the silicate faces. However, the local diffusion coefficients near the middle of the channel are closer to the bulk value for the larger channel widths. These results indicate that reduced porosity values can influence the transport behavior not only by reducing the volume available for transport, but also through alterations of the solute-solvent interactions at smaller scales. 

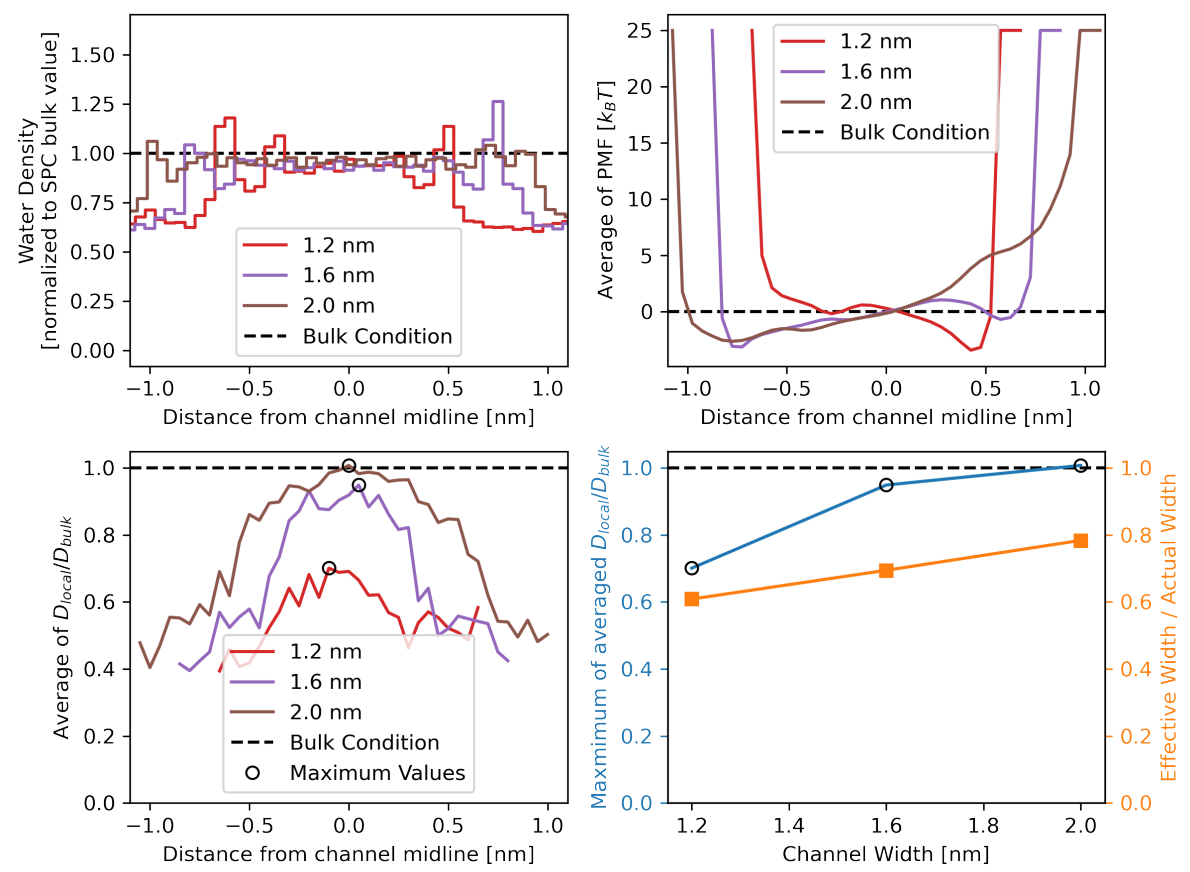

Figure 9: Water density, PMF, and local diffusion coefficient results for channel widths of $1.2,1.6$, and $2.0 \mathrm{~nm}$. These results are averaged over the $y$ direction to show variation across the channel. All channels shown are fully protonated. Top left: water density. The water density appears to oscillate with distance from the silicate face, with the amplitude of the oscillations decreasing with distance. Top right: PMF, with zero as the bulk potential value. Lower left: local diffusion coefficient, normalized to the bulk value. Lower right: maximum values of the normalized local diffusion coefficients for each channel width, and an effective channel width computed from the local diffusion coefficient values. 


\subsubsection{Results for channels with varying surface protonation levels}

To assess the influence of the silicate surface chemistry on transport behavior within the channel, MD simulations were conducted for surface a range of surface protonation levels. Specifically, simulations were conducted with $100 \%, 73 \%$, and $50 \%$ of the silanol groups on the silicate surface protonated. In the deprotonated silanol groups, the hydrogen atom was replaced with a sodium ion that is free to diffuse away from the oxygen ion. These simulations used a channel width of $2.0 \mathrm{~nm}$. Results for water density, PMF, and local diffusion coefficient for these channel widths are shown in Figure 10. The results for water density, PMF, and the local diffusion coefficient are very similar for the three different protonation levels. However, there are subtle differences in the local diffusion coefficient: decreasing levels of protonation seem to be associated with a slight reduction in the local diffusion coefficient values. It is possible that this effect would be more pronounced at smaller channel widths than $2.0 \mathrm{~nm}$.
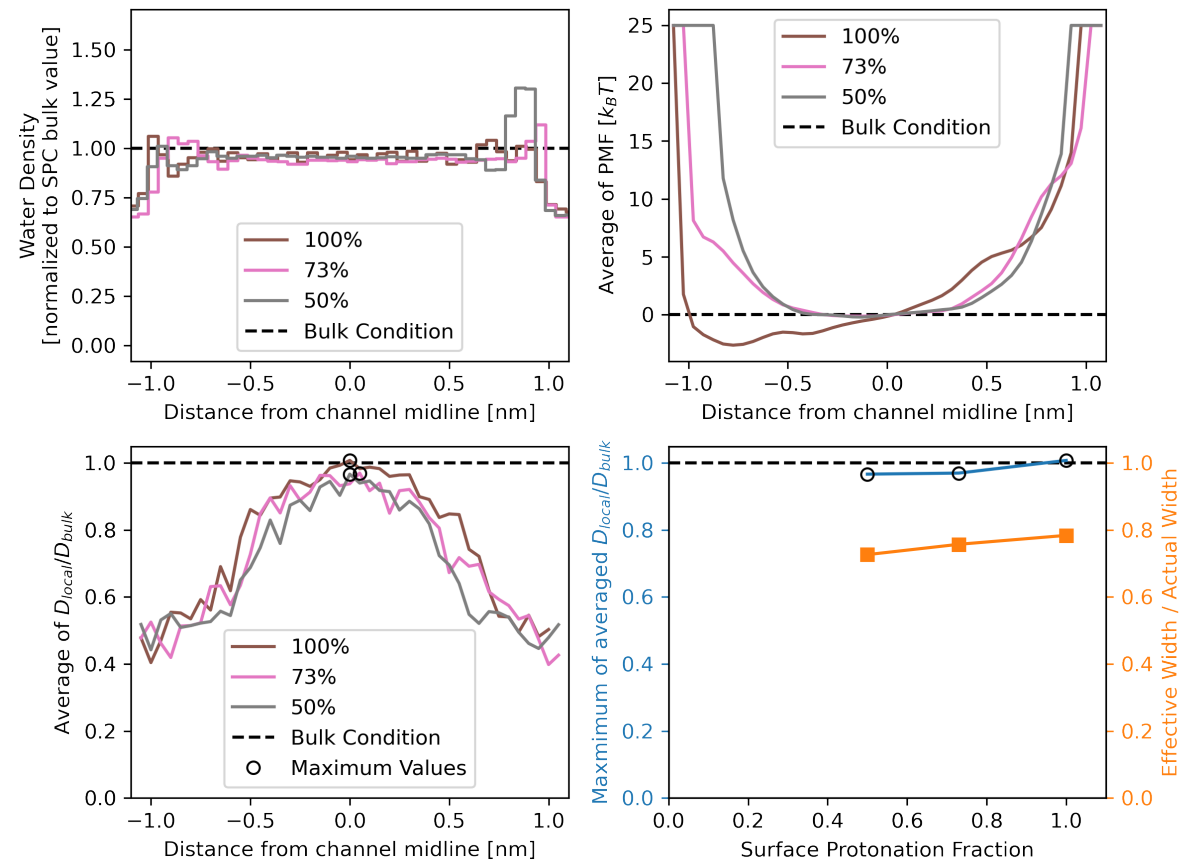

Figure 10: Water density, PMF, and local diffusion coefficient results for surface protonation levels of $100 \%, 73 \%$, and $50 \%$. These results are averaged over the $y$ direction to show variation across the channel. All channels shown are $2.0 \mathrm{~nm}$ in width. Top left: water density. Top right: PMF, with zero as the bulk potential value. Lower left: local diffusion coefficient, normalized to the bulk value. Lower right: maximum values of the normalized local diffusion coefficients for each deprotonation level, and an effective channel width computed from the local diffusion coefficient values. 


\subsection{Homogenization Simulations}

Numerical simulations of the channel were conducted using homogenization theory as described in Section 6.2.2. The results of these simulations are shown in Figure 11. Variations in both the channel width and the surface protonation level, identical to those in the MD simulations, were included in the homogenization calculations. For the condition where the local diffusion coefficient was taken to be spatially invariant in the channel, and identical to the bulk diffusion coefficient, the effective diffusion coefficient can be calculated directly from the porosity (free volume fraction) of the silicate. The results from the homogenization simulations matched this theoretical calculation. As the porosity increases with increasing channel width, these results form an upward-sloping line in the left panel of Figure 11. This result set is not affected by changes in the surface protonation of the channel surfaces, as neither the local diffusion coefficient nor the PMF are included. Consequently, the corresponding line in the right panel of Figure 11 is horizontal.

The MD results showed suppression of the local diffusion coefficient below its bulk value near the channel walls. Accordingly, the results in Figure 11 using the spatially varying diffusion coefficient from the MD simulations show a suppressed effective diffusion coefficient compared to the case where the local diffusion coefficient is equal to the bulk value at all locations. This is true for the homogenization simulations both with and without a PMF applied. Also the local diffusion coefficients seem to vary not only with the channel width, but also with the surface protonation level, to a lesser extent.

To validate the use of homogenization theory for the case of a spatially varying potential, a quadratic potential was tested for conditions without spatial variation in the local diffusion coefficient. This quadratic potential was selected such that is minimum and maximum values within the channel were in the same range as the maxima and minima of the PMF results from the MD simulations. This required the potential to rise more quickly in smaller channels than in wider ones, just as in the PMF data from the MD simulations. As shown in Figure 11, the homogenization results matched the theoretical calculation for this quadratic potential.

The PMF data from the MD simulations includes both regions of higher and lower value than the bulk condition. These areas then become less and more attractive to the diffusing methane, respectively. Overall, the effect of the attractive regions of the potential can actually accelerate diffusion through the channel. The homogenization results in Figure 11 show such an acceleration of diffusion for all of the fully protonated condtions, but a further depression of the diffusion coefficient for de-protonated cases. 

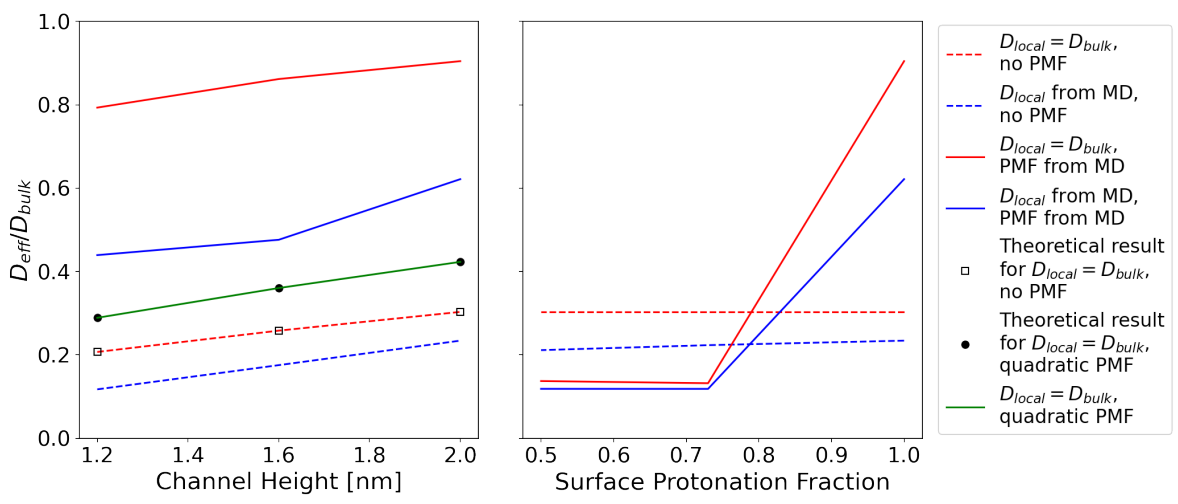

Figure 11: Effective diffusion coefficient predictions from homogenization simulations of the silicate channel. The results are plotted as an effective diffusion coefficient normalized to the bulk diffusion coefficient. Left panel: Results for channels of varying porosity, with all surfaces fully protonated. Right panel: Results for channels of $2.0 \mathrm{~nm}$ width, with different levels of surface protonation. Results from homogenization simulations are shown with continuous lines, while analytically-derived results are plotted as points. Red lines are used for cases where the local diffusion coefficient is spatially invariant and equal to the bulk diffusion coefficient. Blue lines are used for cases where the local diffusion coefficient was taken from the MD results. The green line represents homogenization simulations conducted with a quadratic potential as described in the text, for comparison with an analytical solution for that case. Dashed lines are used for cases without a PMF. Solid lines are used for cases where the PMF was taken from the MD results. 


\section{Conclusions}

The transport behavior of aqueous methane through porous catalysts may play a crucial role in the future design of gas-to-liquid methane conversion processes. With respect to the Langevin dynamics governing such transport behavior, this work has demonstrated that the forces involved in diffusion can be resolved using MD simulations, by measuring the diffusion coefficient and the PMF. The techniques employed herein can be applied even when both of these parameters have spatial variations. Using homogenization simulations, these spatial variables can then be used to obtain the effective transport property for a continuum approximation of the porous medium.

As noted in the results above, changes in the material porosity and the surface chemistry of the pores can have effects on the local diffusion coefficient and PMF within the pore. These changes ultimately impact the effective diffusion coefficient as well, allowing for the tuning of transport properties by the proper selection of material porosity and surface chemistry.

\section{Theory}

\subsection{Hypothesis for the influence of solvent density on local transport behavior}

The motion of a single methane molecule surrounded by a solvent such as water can be described by a Langevin equation in the limit of strong friction:

$$
\xi \dot{x}=-\frac{\partial}{\partial x} V+f(t)
$$

where $\xi$ is the friction coefficient representing solute/solvent interactions, $V$ is a mean force potential, and $f(t)$ is a force varying randomly in time. A single independent variable is shown here for clarity, but the analysis generalizes easily to higher dimensions, as there is no coupling between orthogonal components of the motion. When the solvent is confined to nano-scale pores within a medium such as a silicate, the surface chemistry of the pores could cause local variations in the number density of the solvent molecules. Our expectation is that these local variations in the solvent density will alter the local transport properties of the methane. We describe these effects by making the friction and potential terms of the Langevin equation spatial variables.

Through the Einstein relation between $\xi$ and the diffusion coefficient, $D$, spatial variations in the friction coefficient will produce spatial variations in $D$ as well. This spatial variation was measured in the MD simulations using the approach described in Section 5.2. The spatially varying PMF was measured using standard procedures described in Section 6.1.2.

\subsection{Determination of local diffusion of coefficient from MD simulation}

The diffusion coefficient relates the flux of a diffusion species to its concentration gradient. In homogeneous media the average diffusion can be estimated based on the MSD of individual molecules:

$$
D=\frac{1}{6} \frac{\partial\left\langle r^{2}\right\rangle}{\partial t}
$$

This approach is not applicable to non-homogeneous media, as the MSD would be influenced by the local diffusion coefficient in all the areas visited by the 
diffusing molecule. Instead, the spatial variation of the transport coefficient can be obtained from a Green-Kubo relation. In our model, MD simulations (described in Sect. 6.1) were used to predict Eq. 14 parameters using an approach described in Daldrop, Kowalik, and Netz [11], based on earlier work in Hummer [13] and Woolf and Roux [14]. We also previously used this approach in Setny et al. [15]. The self-diffusion coefficient for the solute methane here implicitly reflects the solute/solvent (water) interactions. Namely, the motion of methane is approximated as over-damped Brownian motion.

To obtain local values of the diffusion coefficient, the methane is restrained by a harmonic potential $\frac{1}{2} K x^{2}$. The equation of motion for the methane molecule is therefore the Langevin equation of Eq. 3.

$$
\xi \dot{x}=-K x+f(t)
$$

The statistical properties of the random variation of $f(t)$ are such that they satisfy Eq. 4 [16-18].

$$
\begin{aligned}
\langle f(t)\rangle & =0 \\
\langle f(0) f(t)\rangle & =2 \xi k_{B} T
\end{aligned}
$$

Under these conditions, it can be shown that the solution for $x(t)$ will have the properties shown in Eq. 5 .

$$
\begin{aligned}
\left\langle x^{2}\right\rangle & =\frac{k_{B} T}{K} \\
\langle x(0) x(t)\rangle & =\left\langle x^{2}\right\rangle e^{-t / \tau}
\end{aligned}
$$

where $\tau=\xi / K$. From these results, the integral of the position autocorrelation function is given by Eq. 6 .

$$
\tau=\frac{1}{\left\langle x^{2}\right\rangle} \int_{0}^{\infty}\langle x(0) x(t)\rangle d t
$$

This allows for determination of the friction coefficient $\xi$, and thus also the local diffusion coefficient $D$, using the Einstein relation of Eq. 7.

$$
D=\frac{k_{B} T}{\xi}=\frac{\left\langle x^{2}\right\rangle}{\tau}
$$

In Eq. 7, the actual MSD value, $\left\langle x^{2}\right\rangle$, is used in place of its theoretical value for a harmonic potential, because the actual potential is the superposition of the harmonic biasing potential and the PMF. The procedure is adopted here, under the assumption that the gradient of the PMF will be small in comparison to the value of $K$ for the restraining potential. Note that larger values of $K$ will confine the methane to a smaller region around the center of the harmonic potential, allowing spatial variations in the local diffusion coefficient to be measured with greater spatial precision. However, large values of $K$ can also invalidate the assumption of over-damping. This can result in oscillatory behavior in the position ACF, making integration more difficult [11]. Here, the value of $K$ for the harmonic potential was selected with the aim of being small enough to maintain over-damped behavior, while remaining larger than reasonable magnitudes for the PMF gradient.

The center of the harmonic restraining potential can be shifted to obtain the local diffusion coefficient at selected spatial locations.

For anisotropic conditions, this analysis can be repeated for each component of the position autocorrelation function to obtain the diagonal matrix components. The simple geometry of the silicate channel studied here makes it very likely that the selected coordinate system is a principal coordinate system, where 
the off-diagonal terms of the matrix are zero. Future applications of this method may involve porous materials with more complex pore geometry, where the offdiagonal terms may be nonzero in some locations. A relatively minor extension of the method for such circumstances would be to rotate the coordinate system of the methane trajectories prior to calculation of the $\mathrm{ACF}$, allowing the diagonal matrix terms to be computed in a different coordinate system. An estimate of the off-diagonal terms could then be obtained from the known transformation matrix between these two coordinate systems.

\section{Methods}

\subsection{Molecular Dynamics}

\subsubsection{Parameterization and simulation}

Molecular dynamics simulations were conducted in GROMACS. The OPLS-AA SPC water model was used for the explicit solvent. The united atom approximation was selected for the methane molecule, on the basis of the results presented in Bhatia and Nicholson [19]. The nonbonded force field parameters for methane were taken from Jorgensen, Madura, and Swenson [20], which used the same functional form for the 12-6 Lennard-Jones potential as GROMACS, so that only unit conversions of the parameters were required. While the combining rule noted in Jorgensen, Madura, and Swenson [20] is the geometric average, the arithmetic average is used herein. The force field parameters for silica were taken from Emami et al. [7, 8], and converted to the units and functional form used in GROMACS. Specifically, the functional form of the 12-6 Lennard-Jones potential for nonbonded interactions in Emami et al. [7] is given by Equation 8, while the functional form used by GROMACS is shown in Equation 9. In both equations, $E$ is the interaction energy, and $r$ is the separation distance between the two atoms. The two functional forms can be used to represent the same interaction potential by computing the GROMACS parameters from the parameters provided by Emami et al. [7] using the relationships of Equation 10.

$$
\begin{gathered}
E=\epsilon_{\text {amber }}\left(\left(\frac{\sigma_{\text {amber }}}{r}\right)^{12}-2\left(\frac{\sigma_{\text {amber }}}{r}\right)^{6}\right) \\
E=4 \epsilon_{\text {gromacs }}\left(\left(\frac{\sigma_{\text {gromacs }}}{r}\right)^{12}-\left(\frac{\sigma_{\text {gromacs }}}{r}\right)^{6}\right) \\
\epsilon_{\text {gromacs }}=\epsilon_{\text {amber }} \\
\sigma_{\text {gromacs }}=2^{-1 / 6} \sigma_{\text {amber }}
\end{gathered}
$$

The nonbonded interaction parameters used in the simulations are listed in Table 3 .

To confirm the proper conversion of the nonbonded force field parameters, the same method was used to convert the parameters from an AMBER input file to the corresponding GROMACS input file parameters for an AMBER force field. This comparison involves an additional step, because the AMBER force field parameters specify the van der Waals radius of each atom, and the depth of the potential well, rather than specifying $\sigma_{\text {amber }}$ and $\epsilon_{\text {amber }}$ directly. The additional calculation is relatively simple, because the functional form of Equation 8 used by AMBER has a well depth that is equal to $\epsilon_{\text {amber, }}$, with the point of minimum potential energy located at $r=\sigma_{\text {amber. }}$. At this minimum, the separation distance $r$ between two identical atoms would be twice the van der Waals radius. Thus,

$\sigma_{\text {amber }}$ is simply twice the specified van der Waals radius, and $\epsilon_{\text {amber }}$ is equal to 


\begin{tabular}{ccccc}
\hline Species & $\sigma[\mathrm{nm}]$ & $\epsilon[\mathrm{kJ} / \mathrm{mole}]$ & $q[\mathrm{e}]$ & Reference \\
\hline $\mathrm{Si}$ & 0.37 & 0.39 & +1.1 & Emami et al. [21] \\
$\mathrm{O}_{\mathrm{Si}}$ & 0.31 & 0.23 & -0.55 & Emami et al. [21] \\
$\mathrm{O}_{\mathrm{H}}$ & 0.31 & 0.51 & -0.68 & Emami et al. [21] \\
$\mathrm{H}$ & 0.096 & 0.063 & +0.4 & Emami et al. [21] \\
$\mathrm{CH}_{4}$ & 0.37 & 1.23 & 0.0 & Jorgensen, Madura, and Swenson [22] \\
\hline
\end{tabular}

Table 3: Non-bonded interaction parameters used for molecular dynamics simulations in GROMACS.

the depth of the potential well. Using this information, the gromacs parameters for the AMBER96 and AMBER99 force fields were successfully reproduced.

Following solvation, energy minimization was performed for 50,000 steps, and then velocities were randomly assigned according to a Maxwell distribution. The system was equilibrated in the Number, Volume, Temperature thermodynamic ensemble (NVT) ensemble for 0.1 nanoseconds, using the Nosé-Hoover thermostat. This was followed by 0.1 nanoseconds of equilibration in the Number, Pressure, Temperature thermodynamic ensemble (NPT) ensemble, using the Berendsen barostat and modified Berendsen thermostat. Production runs followed, using the NVT ensemble with the Nosé-Hoover thermostat. The production runs had a duration of 2 nanoseconds, except where noted otherwise. In all analysis steps, electrostatic interactions were evaluated using the PME method. The dynamic runs used a time-step of 2 femtoseconds, with hydrogen bonds converted to constraints maintained by the LINCS algorithm.

The harmonic restraining potential applied to the methane molecule used a $K$ of $3.00 \times 10^{2} \mathrm{~kJ} \mathrm{~mol}^{-1} \mathrm{~nm}^{-2}$, for each direction. To prevent global translations, rotations, or deformations of the silicate bodies within the simulation unit cell, each atom of the silicate material was placed in a harmonic restraining potential with $K$ of $1.000 \times 10^{3} \mathrm{~kJ} \mathrm{~mol}^{-1} \mathrm{~nm}^{-2}$, for each direction. This includes the hydrogen atoms on the surficial silanol groups, which have affected the solvent-surface interactions.

\subsubsection{Processing methods}

Simulations of the solvated silicate channel without methane were used to calculate the spatial variation of water density within the channel. Water density was calculated from the water molecule trajectories using the MDAnalysispackage [23, 24] (which uses numpy [25]). The production runs for the water density calculations were 128 nanoseconds in duration.

Based on the water density results, portions of the channel were selected for detailed measurement of the local diffusion coefficient and PMF for methane. An $x$-plane was chosen, with dimensions in the $z$-direction fully extending across the channel from one silicate face to the other. The selected area also extended 0.6 nanometers in the $y$-direction. A different region was selected for each channel geometry and deprotonation level. The regions were chosen so as to include areas of both high and low water density at the channel boundaries.

PMF values were computed using the two-dimensional version of WHAM [26] (Version 2.0.10.1), which implements the Weighted Histogram Analysis Method (WHAM) [27-29]. The $y$ and $z$ coordinates of the methane or sodium molecule were used as the reaction coordinates in this approach.

The PMF values resulting from WHAM are relative values, with the minimum value in the data set originally chosen as zero. Instead, the analysis 
conducted here requires that a PMF value of zero represent the conditions of bulk water. Assuming that all studied channels are sufficiently wide that bulk behavior is recovered in the middle of the channel, this location can be used as a reference value for the PMF. After generation by WHAM, the values were shifted such that the average of the PMF values at the middle of the channel would be zero. Also, numerical difficulties were encountered in the homogenization simulations when using the PMF values directly from the MD simulations, even after adjusting to the gauge condition. To allow the simulations to complete successfully, the average value of the PMF was taken in the $y$-direction, such that the PMF used in the homogenization simulations varied only in $z$, which is the direction perpendicular to the silicate faces.

Local diffusion coefficients were calculated using the approach described in Sect. 5.2. Position ACFs were calculated by GROMACS, which returns the ACF function normalized by the MSD as illustrated in Equation 11.

$$
\text { normalized } \operatorname{ACF}(t)=\frac{1}{\left\langle x^{2}\right\rangle}\langle x(0) x(t)\rangle
$$

Observations of typical normalized ACF results showed that, in addition to the exponential decay predicted by Equation 5, there was also an short-duration Gaussian superimposed on the ACF for small values of time. Accordingly, the functional form in Equation 12 was selected as being a generally representative form, with fitting constants $A, \alpha$, and $\sigma$. The analytical integral of this form is shown in Equation 13.

$$
\begin{gathered}
\text { normalized } \mathrm{ACF}_{\text {fit }}(t)=A e^{-t / \sigma}+(1-A) e^{-\alpha t^{2}} \\
\left.\tau=\int_{0}^{\infty} d t \text { (normalized } \mathrm{ACF}_{\text {fit }}(t)\right)=A \sigma+\frac{1-A}{2} \sqrt{\frac{\pi}{\alpha}}
\end{gathered}
$$

Following calculation of a normalized ACF from methane trajectory data, a python script using the numpy package [25] fit the analytical function of Equation 12 to the normalized ACF data. The analytical integration of the normalized ACF was then calculated from Equation 13. To confirm a successful fit, the integral of the normalized ACF was also obtained numerically using the trapezoidal rule over the first 25 picoseconds. Cases where the numerical and analytical integrations differed by more than $25 \%$ were discarded from the data set.

The diffusion coefficients computed from the methane ACFs were generally consistent for the $x$ and $y$ directions, but different in the $z$ direction. Ultimately, the effective diffusion coefficient for only the $x$ and $y$ directions is desired, as the $z$ direction would be impervious. Furthermore, the channel itself is identical in the $x$ and $y$ directions, so the expected results for these two directions would be identical. Accordingly, the homogenization process used an isotropic local diffusion coefficient, which was computed as the arithmetic average of the $x$ and $y$ results from the MD simulations.

As noted in Section 3.1, the local diffusion coefficient results from MD were normalized to the bulk value predicted by the same method. In any cases where the normalized result was greater than 2.0, the result was limited to this value.

Homogenization theory is intended for application to periodic functions. Because the local diffusion coefficients and PMF values from the MD simulations were not periodic, they were reflected about both their right and upper boundaries to produce a symmetric unit cell for homogenization. 


\subsection{Finite Element Modeling}

\subsubsection{Continuum level modeling of aqueous methane diffusion in sil- ica material}

Diffusion is represented in the continuum model by Fick's law [30] which can be written as

$$
\frac{\partial c}{\partial t}=\frac{\partial}{\partial x_{i}} D_{i j} \frac{\partial c}{\partial x_{j}}
$$

where

- $c$ represents the concentration of methane as a function of space and time

- $t$ represents time

- $x_{i}$ represents one of the three spatial coordinates

- the summation convention is used (a repeated index in any term implies summation over all three dimensions for that index)

- $D_{i j}$ represents the diffusion matrix, with three rows and three columns

When the diffusive medium is isotropic and spatially invariant, the diffusion matrix is simply the identity matrix scaled by a diffusion constant, $D$, and Fick's law can be written as

$$
\frac{\partial c}{\partial t}=D \nabla^{2} c
$$

Eq. 15 can be generalized to reflect the influence of a time-independent and spatially-heterogeneous mean field, $V(x)$, using the Smoluchowski equation

$$
\frac{\partial c}{\partial t}=\nabla \cdot\left(D e^{-\frac{V}{k_{B} T}} \nabla\left(c e^{\frac{V}{k_{B} T}}\right)\right)
$$

This can be written in the Slotboom formulation as

$$
\frac{\partial c}{\partial t}=\nabla \cdot(\bar{D} \nabla(\bar{c}))
$$

where

$$
\begin{aligned}
\bar{D} & =D e^{-\frac{V}{{ }^{k} B^{T}}} \\
\bar{c} & =c e^{\frac{V}{k T}}
\end{aligned}
$$

Following this transformation, the steady-state diffusion equation has the same form as the Fickian diffusion equation, with a spatially varying diffusion coefficient.

\subsubsection{Homogenized model of continuum and atomistic scale methane diffusion}

Homogenization [3, 4] is a procedure for separating scales in a partial differential equation for a periodic geometry. The equation used as an input to the procedure describes the processes that takes place at the smaller scale. The procedure produces an equation appropriate for analysis at the larger scale, which contains a parameter found by integrating over the unit cell at the smaller scale. For the homogenization approach to be valid, the relevant length scales must be well-separated. For notational clarity, we denote spatial coordinates at the larger scale by $x_{i}$ and at the smaller scale by $y_{i}$. The volume of the unit cell is denoted as $|Y|$. 
Using the procedure described in Chapter 1 of Bensoussan, Lions, and Papanicolaou [4], the homogenization of Fick's law indicates that the diffusion matrix for the larger scale is:

$$
D_{i j}^{\text {macro }}=\frac{1}{|Y|}\left(\int_{Y}\left(D_{i j}-D_{i k} \frac{\partial \chi_{j}}{\partial y_{k}}\right) d^{n} y\right)
$$

where the vector $\chi$ is defined as the solution to

$$
\frac{\partial}{\partial y_{i}}\left(D_{i k} \frac{\partial \chi_{j}}{\partial y_{k}}\right)=\frac{\partial}{\partial y_{i}} D_{i j}
$$

Homogenization of the Smoluchowski equation was accomplished by using the Slotboom transformation, followed by using the homogenized Fickian equation with a spatially varying diffusion coefficient. In this case, the integral over the unit cell provides a value of $\bar{D}$ for the larger scale. For the inverse Slotboom transformation to obtain the Fickian diffusion coefficient, $D$, for the larger scale, the potential to be used is a constant value representing the potential of the surroundings at the larger scale. This potential must use the same gauge condition as the smaller-scale potential.

\subsubsection{Computational Details}

The finite element meshes for homogenization were created in GMSH [31]. The Finite Element Method (FEM) analysis was conducting with python code relying on version 2019.1.0 of the FEniCS package [32, 33].

In homogenization, the boundary conditions for the unit cell are that the corrector function should be periodic, with period matching the unit cell [4]. The python scripts using FEniCS were able to implement periodic boundary conditions for two-dimensional problems. While FEniCS supports periodic boundary conditions in three-dimensions, the programming effort required is considerably greater. Consequently, periodic boundary conditions were implemented for twodimensional unit cells only. For three-dimensional unit cells, Dirichlet boundary conditions were used instead, with the corrector set to zero at the boundaries. While this is technically a periodic condition, it may be too restrictive for the corrector functions in some cases. Generally, this boundary condition is appropriate for situations where barriers to diffusion within the unit cell are located away from the cell boundaries.

All code written in support of this publication are publicly available at https://bitbucket.org/pkhlab/pkh-lab-analyses. Simulation input files and generate data are available upon request. 


\section{Supplement}

\section{S Acknowledgements}

This work was supported by the Petroleum Research Fund of the American Chemical Society. The authors are also grateful to Markus Schmuck for helpful conversations about homogenization theory.

\section{References}

[1] J. Bear. Dynamics of fluids in porous media. Environmental science series (New York, 1972- ). New York: American Elsevier Pub. Co, 1972. ISBN: 978-0-444-00114-6.

[2] F. A. Coutelieris and J. M. P. Q. Delgado. Transport processes in porous media. en. Advanced structured materials v.20. OCLC: ocn769420091. Heidelberg ; New York: Springer, 2012. ISBN: 978-3-642-27909-6.

[3] J.-L. Auriault, C. Boutin, and C. Geindreau. Homogenization of coupled phenomena in heterogenous media. eng. London, UK; Hoboken, N.J: ISTE; J. Wiley, 2009. ISBN: 978-1-84821-161-2.

[4] A. Bensoussan, J.-L. Lions, and G. Papanicolaou. Asymptotic analysis for periodic structures. Providence, R.I: American Mathematical Society, 2011. ISBN: 978-0-8218-5324-5.

[5] N. Hansen et al. "Analysis of Diffusion Limitation in the Alkylation of Benzene over H-ZSM-5 by Combining Quantum Chemical Calculations, Molecular Simulations, and a Continuum Approach". en. In: The Journal of Physical Chemistry C 113.1 (Jan. 2009), pp. 235-246. ISSN: 1932-7447, 1932-7455.

[6] T. Bui et al. "Transport Mechanism of Guest Methane in Water-Filled Nanopores". en. In: The Journal of Physical Chemistry C 121.29 (July 2017), pp. 15675-15686. ISSN: 1932-7447, 1932-7455.

[7] F. S. Emami et al. "Force Field and a Surface Model Database for Silica to Simulate Interfacial Properties in Atomic Resolution". en. In: Chemistry of Materials 26.8 (Apr. 2014), pp. 2647-2658. ISSN: 0897-4756, 1520-5002.

[8] F. S. Emami et al. "Correction to Force Field and a Surface Model Database for Silica to Simulate Interfacial Properties in Atomic Resolution". en. In: Chemistry of Materials 28.1 (Jan. 2016), pp. 406-407. ISSN: 0897-4756, 1520-5002.

[9] P. A. Witherspoon and D. N. Saraf. "Diffusion of Methane, Ethane, Propane, and n-Butane in Water from 25 to $43^{\circ}$ ". en. In: The Journal of Physical Chemistry 69.11 (Nov. 1965), pp. 3752-3755. ISSN: 0022-3654, 1541-5740.

[10] C. Oostenbrink et al. "A biomolecular force field based on the free enthalpy of hydration and solvation: The GROMOS force-field parameter sets 53A5 and 53A6". en. In: Journal of Computational Chemistry 25.13 (Oct. 2004), pp. 1656-1676. ISSN: 0192-8651, 1096-987X.

[11] J. O. Daldrop, B. G. Kowalik, and R. R. Netz. "External Potential Modifies Friction of Molecular Solutes in Water". en. In: Physical Review X 7.4 (Dec. 2017). ISSN: 2160-3308.

[12] D. Frenkel and B. Smit. Understanding molecular simulation: from algorithms to applications. 2nd ed. Computational science series 1. San Diego: Academic Press, 2002. ISBN: 978-0-12-267351-1. 
[13] G. Hummer. "Position-dependent diffusion coefficients and free energies from Bayesian analysis of equilibrium and replica molecular dynamics simulations". en. In: New Journal of Physics 7 (Feb. 2005), pp. 34-34. ISSN: 1367-2630.

[14] T. B. Woolf and B. Roux. "Conformational Flexibility of o-Phosphorylcholine and o-Phosphorylethanolamine: A Molecular Dynamics Study of Solvation Effects". en. In: Journal of the American Chemical Society 116.13 (June 1994), pp. 5916-5926. ISSN: 0002-7863.

[15] P. Setny et al. "Solvent fluctuations in hydrophobic cavity-ligand binding kinetics". en. In: Proceedings of the National Academy of Sciences 110.4 (Jan. 2013), pp. 1197-1202. ISSN: 0027-8424, 1091-6490.

[16] R. Zwanzig. Nonequilibrium statistical mechanics. Oxford University Press, 2001.

[17] F. Reif. Fundamentals of statistical and thermal physics. eng. reiss. OCLC: 845506926. Long Grove, Ill: Waveland Press, 2009. ISBN: 978-1-57766-6127.

[18] R. K. Pathria and P. D. Beale. Statistical mechanics. 3rd ed. Amsterdam ; Boston: Elsevier/Academic Press, 2011. ISBN: 978-0-12-382188-1.

[19] S. K. Bhatia and D. Nicholson. "Adsorption and Diffusion of Methane in Silica Nanopores: A Comparison of Single-Site and Five-Site Models". en. In: The Journal of Physical Chemistry C 116.3 (Jan. 2012), pp. 23442355. ISSN: 1932-7447, 1932-7455.

[20] W. L. Jorgensen, J. D. Madura, and C. J. Swenson. "Optimized intermolecular potential functions for liquid hydrocarbons". en. In: Journal of the American Chemical Society 106.22 (Oct. 1984), pp. 6638-6646. ISSN: 0002-7863.

[21] F. S. Emami et al. "Force Field and a Surface Model Database for Silica to Simulate Interfacial Properties in Atomic Resolution". In: Chemistry of Materials 26.8 (2014), pp. 2647-2658.

[22] W. L. Jorgensen, J. D. Madura, and C. J. Swenson. "Optimized intermolecular potential functions for liquid hydrocarbons". In: Journal of the American Chemical Society 106.22 (1984), pp. 6638-6646.

[23] R. Gowers et al. "MDAnalysis: A Python Package for the Rapid Analysis of Molecular Dynamics Simulations". In: Proceedings of the 15th Python in Science Conference. SciPy, 2016.

[24] N. Michaud-Agrawal et al. "MDAnalysis: A toolkit for the analysis of molecular dynamics simulations". In: Journal of Computational Chemistry 32.10 (Apr. 2011). Publisher: Wiley, pp. 2319-2327.

[25] S. Van Der Walt, S. C. Colbert, and G. Varoquaux. "The NumPy array: a structure for efficient numerical computation". In: Computing in Science \&6 Engineering 13.2 (2011). Publisher: AIP Publishing, pp. 22-30.

[26] A. Grossfield. WHAM: an implementation of the weighted histogram analysis method, Version 2.0.10. 2005.

[27] S. Kumar et al. "THE weighted histogram analysis method for free-energy calculations on biomolecules. I. The method". en. In: Journal of Computational Chemistry 13.8 (Oct. 1992), pp. 1011-1021. ISSN: 0192-8651, 1096-987X. 
[28] S. Kumar et al. "Multidimensional free-energy calculations using the weighted histogram analysis method". en. In: Journal of Computational Chemistry 16.11 (Nov. 1995), pp. 1339-1350. ISSN: 0192-8651, 1096-987X.

[29] B. Roux. "The calculation of the potential of mean force using computer simulations". en. In: Computer Physics Communications 91.1-3 (Sept. 1995), pp. 275-282. ISSN: 00104655.

[30] J. Crank. Mathematics of Diffusion. Oxford: Clarendon Press, 1970.

[31] C. Geuzaine and J.-F. Remacle. "Gmsh: A 3-D finite element mesh generator with built-in pre- and post-processing facilities: THE GMSH PAPER". en. In: International Journal for Numerical Methods in Engineering 79.11 (Sept. 2009), pp. 1309-1331. ISSN: 00295981.

[32] M. S. Alnæs et al. "The FEniCS Project Version 1.5". In: Archive of Numerical Software 3.100 (2015).

[33] A. Logg, G. N. Wells, and J. Hake. "DOLFIN: a C++/Python Finite Element Library". In: Automated Solution of Differential Equations by the Finite Element Method, Volume 84 of Lecture Notes in Computational Science and Engineering. Ed. by A. Logg, K.-A. Mardal, and G. N. Wells. Springer, 2012. Chap. 10. 
Supplementary Information (SI) 